\title{
A tale of two $\alpha$-tubulin tails
}

Victoria J Allan

\begin{abstract}
Post-translational modifications of tubulin, such as the removal of the C-terminal tyrosine of $\alpha$-tubulin, have long been proposed to influence the ability of microtubule motors to walk along the microtubule surface. This hypothesis has now been tested for cytoplasmic dynein-1 (dynein), revealing that active dynein-dynactinadaptor complexes prefer to start moving on tyrosinated microtubules. This choice is governed by the $\mathrm{p} 150$ subunit of dynactin. Once moving, however, dynein is not choosy about whether the microtubule is tyrosinated or not.
\end{abstract}

See also: RJ McKenney et al (June 2016)

M icrotubules (MTs) provide the tracks along which the dynein and kinesin families of motors move. Together, they generate the myriad complex movements that are needed for cells to grow, divide, move and communicate. MTs are polymers of alpha and beta tubulin dimers (Fig 1), which are very highly conserved eukaryotic proteins. In the cell, MTs vary greatly in their longevity, with some lasting only minutes, while others persist for many hours (Yu et al, 2015). MTs are particularly stable within axons, cilia and flagella. Such stable MTs accumulate a range of post-translational modifications (PTMs), including acetylation, polyamination, polyglutamylation, polyglycylation and the removal of C-terminal tyrosine from $\alpha$-tubulin (Janke, 2014; Yu et al, 2015). However, many cultured cell types, such as HeLa, have mostly unmodified tubulin.

Several of these PTMs take place on the many glutamates in the unstructured C-terminal tails (CTTs) of $\alpha$ - and $\beta$-tubulin, which extend out from the MT lattice into the cytoplasm (Fig 1). Here, they are ideally placed to influence the interaction between the MT and its motor proteins and are known to influence the activity of a number of kinesins (Janke, 2014; Yu et al, 2015). In vitro motility assays have shown that proteolytic removal of both $\alpha$ - and $\beta$-tubulin CTTs using subtilisin greatly reduced the ability of mammalian cytoplasmic dynein-1 (dynein) to move along MTs (McKenney et al, 2014).

An elegant refinement of this in vitro approach has allowed McKenney and coworkers (McKenney et al, 2016) to investigate how tubulin CTTs and $\alpha$-tubulin tyrosination influence dynein's behaviour. The authors generated yeast tubulins whose CTTs were replaced with mammalian CTTs (Sirajuddin et al, 2014). These were expressed and purified from yeast, allowing the authors to generate MTs of defined composition (Fig 1). They were combined with active tripartite complexes containing pig brain dynein, its accessory complex dynactin and the adaptor protein BicD2. These complexes (DDBs) move highly processively along MTs in vitro, whereas dynein alone, or dynein mixed with dynactin, does not (McKenney et al, 2014; Schlager et al, 2014). Complex formation is thought to cause structural rearrangements that activate dynein and expose the MT binding domain of dynactin's p150 subunit (Carter et al, 2016).

The absence of the $\alpha$-tubulin CTT greatly decreased the number of DDBs moving processively, whereas the lack of the $\beta$-tubulin CTT had only a small effect. In addition, some DDBs undergo diffusive movements backwards and forwards along MTs (McKenney et al, 2014), and this motion also needed the $\alpha$-tubulin CTT. Using recombinant human dynein heavy chain or a truncation of the neuronal p150 subunit that interacts with MTs via its CAP-Gly and basic domains (Culver-Hanlon et al, 2006; Lazarus et al, 2013), the authors found that binding of p150, but not dynein, was greatly reduced when the $\alpha$-tubulin CTT was missing. This is in keeping with p150's CAP-Gly domain's ability to bind to GEEY/F motifs, one of which is present at the C-terminus of tyrosinated $\alpha$-tubulin.

These data focussed the authors' attention on the $\alpha$-tubulin tyrosination cycle. Newly assembled MTs contain $\alpha$-tubulin with a C-terminal tyrosine. Over time, this tyrosine is slowly cleaved off by an unknown carboxypeptidase, leading to MTs containing $\alpha$-tubulin with a C-terminal glutamate (Janke, 2014; Yu et al, 2015). Most MTs disassemble before this happens, so these "Glu MTs" are a stable population. Once they disassemble, tyrosine is added back to $\alpha$-tubulin by tubulin tyrosine ligase, ready for another round of polymerisation (Janke, 2014; Yu et al, 2015).

This cycle is lacking from yeast (as are other PTMs), and so McKenney et al (2016) could purify yeast $\alpha$-tubulin with a mammalian CTT terminating in either glutamate or tyrosine, and generate tyrosinated or Glu MTs. DDB complexes were four times more likely to move on tyrosinated than Glu MTs. Dynein heavy chain alone bound to either type, whereas p150 had a strong preference for tyrosinated $\alpha$-tubulin. Similar results were seen using MTs assembled from carboxypeptidase A-treated pig brain tubulin, which lacks the C-terminal tyrosine, but still has other PTMs. This suggested that it was p150, not dynein, that specified DDB's preference for tyrosinated MTs. Further analysis showed that p150's CAP-Gly domain was responsible, as a construct lacking this domain (mimicking the naturally occurring p135 neuronal splice form) had no preference for tyrosinated MTs, although its MT binding affinity was lower, as previously seen (Lazarus et al, 2013). This lower affinity would explain why DDBs made using 

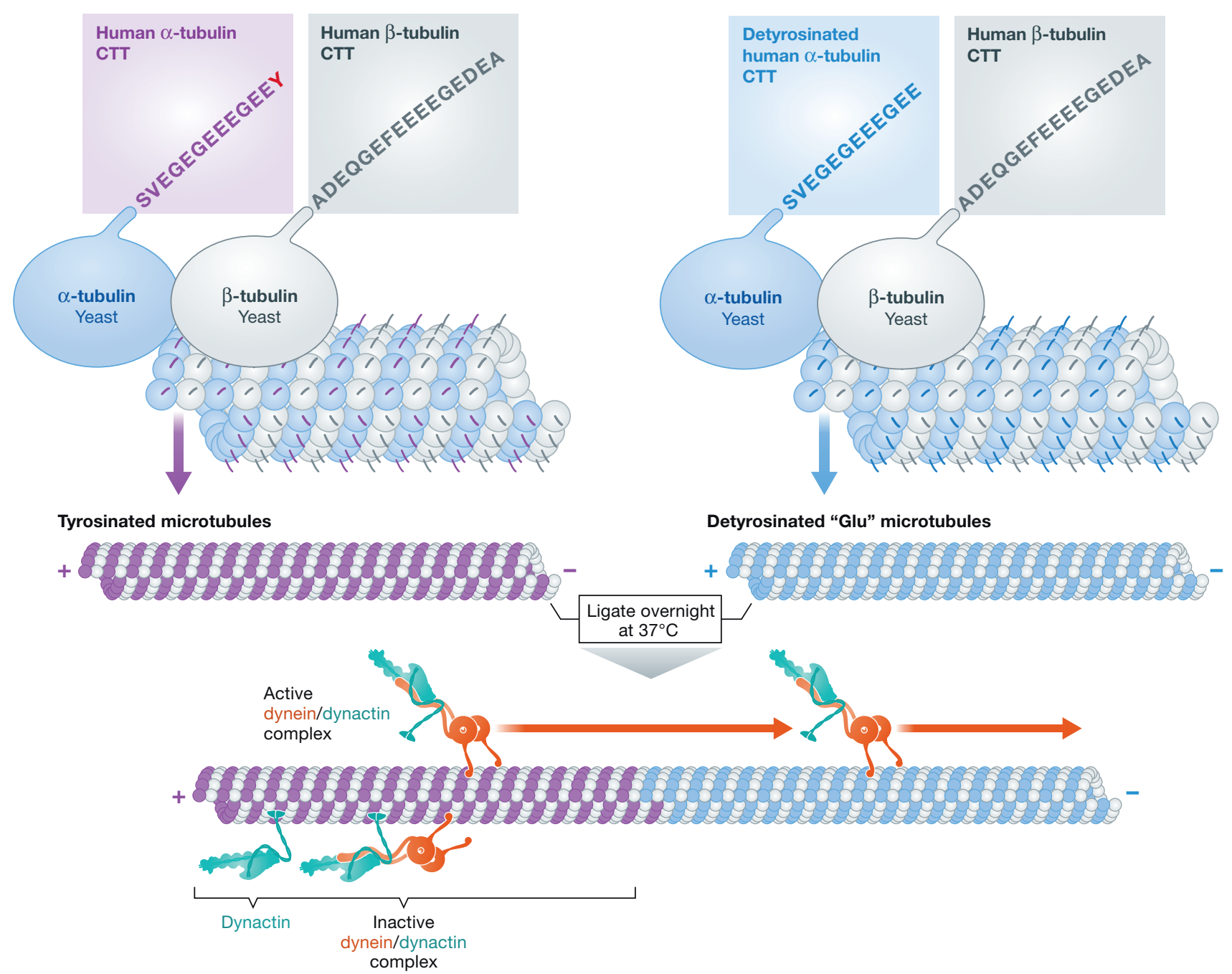

Figure 1. Assembling microtubules with defined C-terminal tails.

Chimeric tubulin proteins consisting of the core of yeast $\alpha$ - and $\beta$-tubulins coupled to specific human CTT sequences allowed McKenney et al (2016) to make microtubules with or without the final $\alpha$-tubulin tyrosine. Microtubules incubated overnight will join end-to-end, generating microtubules that are part tyrosinated, part detyrosinated ("Clu"). Dynactin and inactive dynein-dynactin complexes shun the Glu-microtubule segments, whereas active dynein-dynactin complexes will move on either microtubule type.

p135 dynactin were 3 times less likely to start moving than DDBs with full-length p150, although the motile properties of each type of complex when moving were identical (McKenney et al, 2014).

So far, so good. However, what would happen in an axon, where MTs in the growth cone are tyrosinated, favouring the p150-mediated engagement of the active dynein complex with the MT, but those in the axon shaft are detyrosinated? Would the dynein-dynactin complexes get started, only to hit less favourable tracks? The authors used a neat trick to address this issue, where Glu and tyrosinated MTs were joined together end-to-end (Fig 1). Although p150 molecules or DDB complexes that were diffusing along tyrosinated MTs always stopped or fell off when they reached a join with a Glu MT, $60 \%$ of actively moving DDBs sailed across the boundary to continue moving along the Glu MT. Similar results were seen when CPA treatment was used to make Glu MTs. Forty per cent of processive DDBs would even continue onto subtilisin-treated MTs, which lack the CTTs of both subunits.

Taken together, the data support a model where the interaction between the p150 CAP-Gly domain and tyrosinated $\alpha$-tubulin brings the dynein-dynactin-adaptor complex into contact with the MT. However, once moving, the complex no longer needs the
p150-MT interaction, and dynein's lack of preference for MT type means that either tyrosinated or Glu MTs will do. This is also supported by recent complementary work from the Holzbaur laboratory (Nirschl et al, 2016). Such a scenario makes sense in the cell, since dynein drives the movement from the cell periphery, where there are many tyrosinated MTs ends, towards the cell centre. This model fits well with in vivo observations showing that the CAP-Gly domain of p150 is needed for an efficient initiation of dynein-driven lysosome movement at neurite tips, but not for motility along the axon shaft (Lloyd et al, 2012; Moughamian \& Holzbaur, 2012). It would 
also allow dynein to carry its cargo over any detyrosinated regions in a single MT.

One open question is how many MTs in vivo contain enough detyrosinated tubulin to affect p150, since DDBs initiated movement equally well on tyrosinated MTs and those containing 50\% detyrosinated $\alpha$-tubulin. In addition, what happens when multiple dynein complexes are interacting with the MT in situations where large amounts of force must be generated? Another issue is whether other tubulin PTMs affect DDB motility or p150-MT interactions, and whether tubulin isotypes have any effect. While the recombinant tubulin methodology pioneered by the authors (Sirajuddin et al, 2014; McKenney et al, 2016) will provide a means of addressing some of these challenges, new in vivo approaches will undoubtedly also be needed.

\section{References}

Carter AP, Diamant AG, Urnavicius L (2016) How dynein an dynactin transport cargos: a structural perspective. Curr Opin Struct Biol 37: $62-70$

Culver-Hanlon T, Lex S, Stephens A, Quintyne N, King $S$ (2006) A microtubule-binding domain in dynactin increases dynein processivity by skating along microtubules. Nat Cell Biol 8: $264-270$

Janke C (2014) The tubulin code: molecular components, readout mechanisms, and functions. J Cell Biol 206: 461-472

Lazarus JE, Moughamian AJ, Tokito MK, Holzbaur EL (2013) Dynactin subunit p150(Glued) is a neuron-specific anti-catastrophe factor. PLOS Biol 11: e1001611

Lloyd T, Machamer C, O’Hara K, Kim J, Collins S, Wong $\mathrm{M}$, Sahin B, Imlach W, Yang Y, Levitan E, McCabe B, Kolodkin A (2012) The p150Glued CAP-Gly domain regulates initiation of retrograde transport at synaptic termini. Neuron 74: 344-360

McKenney R, Huynh W, Tanenbaum M, Bhabha G, Vale RD (2014) Activation of cytoplasmic dynein motility by dynactin-cargo adapter complexes. Science 345: $337-341$
McKenney RJ, Huynh W, Vale RD, Sirajuddin M (2016) Tyrosination of $\alpha$-tubulin controls the initiation of processive dynein-dynactin motility. EMBO J 35: 1175-1185

Moughamian A, Holzbaur ELF (2012) Dynactin is required for transport initiation from the distal axon. Neuron 74: $331-343$

Nirschl J), Magiera MM, Lazarus JE, Janke C, Holzbaur EL (2016) $\alpha$-Tubulin Tyrosination and CLIP-170 Phosphorylation Regulate the Initiation of Dynein-Driven Transport in Neurons. Cell Rep 11: $2637-2652$

Schlager MA, Hoang H, Urnavicius L, Bullock S, Carter AP (2014) In vitro reconstitution of a highly processive recombinant human dynein complex. EMBO J 33: 1855-1868

Sirajuddin M, Rice LM, Vale RD (2014) Regulation of microtubule motors by tubulin isotypes and post-translational modifications. Nat Cell Biol 16: $335-344$

Yu I, Garnham CP, Roll-Mecak A (2015) Writing and Reading the Tubulin Code. J Biol Chem 290 $17163-17172$ 